\title{
Comparison of Clinical Tools for Measurements of Regional Stress and Rest Myocardial Blood Flow Assessed with ${ }^{13}$ N-Ammonia PET/CT
}

\author{
Piotr J. Slomka ${ }^{1}$, Erick Alexanderson ${ }^{2,3}$, Rodrigo Jácome ${ }^{2}$, Moises Jiménez ${ }^{2,3}$, Edgar Romero ${ }^{2}$, Aloha Meave ${ }^{2,3}$, \\ Ludovic Le Meunier ${ }^{4}$, Magnus Dalhbom ${ }^{5}$, Daniel S. Berman ${ }^{1}$, Guido Germano ${ }^{1}$, and Heinrich Schelbert ${ }^{5}$ \\ ${ }^{1}$ Cedars-Sinai Medical Center, Los Angeles, California; ${ }^{2}$ Facultad de Medicina, Universidad Nacional Autónoma de México, Mexico \\ City, Mexico; ${ }^{3}$ Cardiovascular Imaging and Nuclear Medicine Department, Instituto Nacional de Cardiología "Ignacio Chávez," \\ Mexico City, Mexico; ${ }^{4}$ Siemens Healthcare Molecular Imaging, Knoxville, Tennessee; and ${ }^{5}$ University of California Los Angeles, Los \\ Angeles, California
}

Several models for the quantitative analysis of myocardial blood flow (MBF) at stress and rest and myocardial flow reserve (MFR) with ${ }^{13} \mathrm{~N}$-ammonia myocardial perfusion PET have been implemented for clinical use. We aimed to compare quantitative results obtained from 3 software tools (QPET, syngo MBF, and PMOD), which perform PET MBF quantification with either a 2-compartment model (QPET and syngo MBF) or a 1-compartment model (PMOD). Methods: We considered 33 adenosine stress and rest ${ }^{13} \mathrm{~N}$-ammonia studies $(22$ men and 11 women). Average age was $54.5 \pm 15 \mathrm{y}$, and average body mass index was $26 \pm 4.2$. Eighteen patients had a very low likelihood of disease, with no chest pain, normal relative perfusion results, and normal function. All data were obtained on a PET/CT scanner in list mode with CT attenuation maps. Sixteen dynamic frames were reconstructed (twelve 10-s, two 30-s, one 1-min, and one 6-min frames). Global and regional stress and rest MBF and MFR values were obtained with each tool. Left ventricular contours and input function region were obtained automatically in system QPET and syngo MBF and manually in PMOD. Results: The flow values and MFR values were highly correlated among the 3 packages $\left(R^{2}\right.$ ranging from 0.88 to 0.92 for global values and from 0.78 to 0.94 for regional values. Mean reference MFR values were similar for QPET, syngo MBF, and $\operatorname{PMOD}(3.39 \pm 1.22,3.41 \pm 0.76$, and $3.66 \pm 1.19$, respectively) by 1 -way ANOVA $(P=0.74)$. The lowest MFR in very low likelihood patients in any given vascular territory was 2.25 for QPET, 2.13 for syngo MBF, and 2.23 for PMOD. Conclusion: Different implementations of 1- and 2-compartment models demonstrate an excellent correlation in MFR for each vascular territory, with similar mean MFR values.

Key Words: myocardial blood flow; perfusion, $\mathrm{PET} / \mathrm{CT}$; $\mathrm{NH}_{3}$; quantitative analysis

J Nucl Med 2012; 53:171-181

DOI: 10.2967/jnumed.111.095398

Received Jul. 5, 2011; revision accepted Oct. 5, 2011.

For correspondence or reprints contact: Piotr J. Slomka, Cedars-Sinai Medical Center, 8700 Beverly Blvd., Taper \#A047, Los Angeles, CA 90048.

E-mail: piotr.slomka@cshs.org

Published online Jan. 6, 2012.

COPYRIGHT @ 2012 by the Society of Nuclear Medicine, Inc.
$\mathbf{T}$ he analysis of myocardial blood flow (MBF) at stress and rest from PET images is an important tool for clinicians and provides information complementary to relative perfusion analysis (1). Recent advances in PET image reconstruction allow dynamic data, perfusion and gated data to be obtained from the same list-mode file with single injection of the radiopharmaceutical and without additional imaging time (2). For the routine clinical use of dynamic data, robust automated quantitative tools are required. Several such tools are now emerging as add-ons to standard quantitative packages or stand-alone tools (1). In addition, each tool employs different methods of segmenting the left ventricle and sampling the counts in the myocardium and blood pool to obtain input curves. Absolute blood flow results can be obtained rapidly and automatically from PET studies, but little is known about the intercomparison of these new tools. In this study, we aimed to compare 3 approaches that have been clinically implemented for the quantitative analysis of $\mathrm{MBF}$ and myocardial flow reserve (MFR) with ${ }^{13} \mathrm{~N}$-ammonia myocardial perfusion PET. These methods include QPET (Cedars-Sinai), syngo MBF (Siemens Medical Solutions), and PMOD (PMOD Technologies) (3), each of which performs MBF quantification with different implementations of a 1-compartment model (PMOD (3)) or a 2-compartment model (QPET (4) and syngo MBF (5)) and different methods of segmenting the left ventricle and sampling the counts in the myocardium and blood pool. We include in these comparisons reference-limit values for patients with a very low likelihood of coronary artery disease.

\section{MATERIALS AND METHODS}

\section{Patients}

This was a retrospective study of patients referred for myocardial perfusion imaging with ${ }^{13} \mathrm{~N}$-ammonia PET for the evaluation of suspected ischemic disease. We considered 2 consecutive groups of patients: a group with a very low likelihood of coronary artery disease and an ischemic group. Patients in the very 
low likelihood group had less than a 5\% likelihood of coronary artery disease within $10 \mathrm{y}$ as defined by the Framingham Heart Study Group (6) and visually normal relative perfusion results obtained from static data. Patients in the ischemic group had evidence of ischemia on the ammonia static perfusion study (as visually assessed). No correlating invasive angiography information was available for these studies.

Thirty-three (22 men and 11 women) adenosine stress and rest ${ }^{13} \mathrm{~N}$-ammonia studies satisfied these criteria in the 2 groups (18 in the very low likelihood group and 15 in the ischemic group). The average age for participants was $54.6 \pm 14.6 \mathrm{y}$, and the average body mass index was $26 \pm 4.0$. Eighteen of the patients were asymptomatic and had a very low likelihood of coronary artery disease. Patient demographics are presented in Table 1. The hemodynamic information at stress and rest is presented in Table 2.

\section{Data Acquisition}

All images were acquired on a whole-body 64-slice PET/CT scanner (Biograph True Point; Siemens Medical Solutions) in 3dimensional (3D) list mode. Patients were studied after an overnight fast, and all refrained from caffeine-containing beverages or theophylline-containing medications for $24 \mathrm{~h}$ before the study. Myocardial perfusion was assessed at rest and during vasodilator stress with adenosine and ${ }^{13} \mathrm{~N}$-ammonia as a blood flow tracer. Two CT-based transmission scans (140 kVp; 20-30 $\mathrm{mA}$; pitch of 1.35) were obtained before the rest perfusion studies and after the stress perfusion studies, for correction of photon attenuation. The registration of the CT attenuation map with the PET images was verified visually by an experienced technologist, and alignment was corrected if necessary by manual 3D translation. Regional myocardial perfusion was first assessed during rest using $740 \mathrm{MBq}$ of ${ }^{13} \mathrm{~N}$-ammonia. Rest imaging extended for $10 \mathrm{~min}$ and began a few seconds before the ${ }^{13} \mathrm{~N}$-ammonia injection. The ${ }^{13} \mathrm{~N}$ ammonia was administered as a single peripheral intravenous bolus $(3-5 \mathrm{~s})$, followed by a $10-\mathrm{mL}$ saline flush. Thirty minutes later, a pharmacologic stress test was performed, beginning with the injection of adenosine during a 6-min period (140 $\mu \mathrm{g} / \mathrm{kg} / \mathrm{min})$. A second dose of $740 \mathrm{MBq}$ of ${ }^{13} \mathrm{~N}$-ammonia was injected at the third minute of the adenosine infusion. Stress image acquisition was started a few seconds before the radiotracer injection.

All images were acquired in 3D list mode. During both the CT and the PET acquisitions, the patients were instructed to breathe normally. Sixteen dynamic frames were reconstructed (twelve 10-s,

TABLE 1

Patient Characteristics $(n=33)$

\begin{tabular}{lc}
\hline \multicolumn{1}{c}{ Characteristic } & Value \\
\hline Men $(n)$ & $22(66 \%)$ \\
Mean age \pm SD $(y)$ & $54.6 \pm 14.6$ \\
Mean body mass index \pm SD & $26.4 \pm 4.0$ \\
Patients with diabetes $(n)$ & 5 \\
Patients with hypertension $(n)$ & 16 \\
Patients with dyslipidemia $(n)$ & 18 \\
Smokers $(n)$ & 14 \\
Patients with a family history $(n)$ & 13 \\
Patients with myocardial infarction $(n)$ & 9 \\
Very low likelihood patients $(n)$ & 18 \\
\hline
\end{tabular}

TABLE 2

Hemodynamics in Patients

\begin{tabular}{lc}
\hline \multicolumn{1}{c}{ Characteristic } & Value \\
\hline Rest & $67.2 \pm 11.0$ \\
Heart rate (beats/min) & $124.0 \pm 14.9$ \\
Systolic blood pressure $(\mathrm{mm} \mathrm{Hg})$ & $8,405.6 \pm 2,095.9$ \\
RPP (beats/min $\cdot \mathrm{mm} \mathrm{Hg})$ & $78.9 \pm 18.1$ \\
Stress & $124.2 \pm 18.1$ \\
Heart rate (beats/min) & $9,790.7 \pm 2,679.9$ \\
Systolic blood pressure $(\mathrm{mm} \mathrm{Hg})$ & \\
RPP (beats/min $\cdot \mathrm{mm} \mathrm{Hg})$ & \\
& \\
\hline RPP $=$ rate-pressure product. & \\
\hline
\end{tabular}

two 30-s, one 1-min, and one 6-min frames, for a total of $10 \mathrm{~min}$ ). Standard reconstruction (2-dimensional attenuation-weighted ordered-subsets expectation maximization) was used with 3 iterations and 14 subsets and 3D postfiltering with a 5-mm gaussian kernel. Transverse data were reformatted to a $168 \times 168 \times 47$ matrix with 2-mm pixels for each dynamic frame. Late perfusion images were reconstructed using $7 \mathrm{~min}$ of the acquisition after a 2-min delay to allow for blood-pool clearance. The reconstruction parameters were identical to the dynamic reconstruction.

\section{Myocardial Perfusion Analysis}

Two experienced nuclear cardiologists analyzed the perfusion images in standard cardiac orientations using the 17-segment model. Myocardial perfusion was described as normal uptake, mild defect, moderate defect, or severe defect. Moreover, each segment was scored using a 5-point scoring system, where 0 is normal uptake, 1 is mildly reduced uptake, 2 is moderate reduced uptake, 3 is severely reduced uptake, and 4 is absence of radiotracer uptake. For each rest and stress image, the segmental scores were summed to derive the summed rest score and the summed stress score. The scan findings were considered normal if the summed stress score was less than 4, mildly abnormal if between 4 and 7, moderately abnormal if between 8 and 11, and severely abnormal if 12 or more.

\section{MBF Analysis}

Global and regional MBF values in $\mathrm{mL} / \mathrm{g} / \mathrm{min}$ and MFR values were obtained with each tool. Left ventricular (LV) contours and input function region were obtained automatically with minimal operator intervention in QPET and syngo MBF and manually in PMOD. Each tool was analyzed independently by 3 different expert operators ( 1 for each software tool) who were blinded to the MBF quantification results.

QPET Analysis. The cases were processed in batch mode. LV contours were positioned automatically with a recently described improved QPET algorithm (7). The LV contour was determined from the summed dynamic image data, skipping the first $2 \mathrm{~min}$ using the QPET algorithm (7), which is based on the original quantitative gated SPECT contour detection principles (8) but takes into account the better valve definition of attenuation-corrected high-resolution PET. The $3 \mathrm{D}$ region for the $\mathrm{LV}$ input function was automatically placed in the middle of the valve plane and was cylindric with a $1-\mathrm{cm}$ radius and $2-\mathrm{cm}$ length, with its long axis oriented along the long axis of the heart. The dynamic myo- 
cardial samples were obtained from the polar map by analyzing all time frames within the fixed LV contour boundaries.

Kinetic modeling followed the approach proposed by Choi et al. (4). Briefly, a 2-compartment model is used with ${ }^{13} \mathrm{~N}$-ammonia activity in the freely diffusible compartment (vascular and extravascular) and metabolically trapped ${ }^{13} \mathrm{~N}$-ammonia in the second compartment. The conversion of freely diffusible ${ }^{13} \mathrm{~N}$-ammonia to metabolically bound ${ }^{13} \mathrm{~N}$-ammonia glutamine (forward, $K_{1}$ ), the clearance constant of ${ }^{13} \mathrm{~N}$-ammonia, and the spillover fraction from blood to myocardium are computed by numeric optimization. An irreversible model was assumed (no reverse clearance from the metabolically trapped compartment). The distribution volume of free ammonia in the myocardium was assumed to be 0.8 . Other specific assumptions have been previously described (4). Only the first $2 \mathrm{~min}$ of the dynamic acquisition are considered during the curve fitting. No metabolite correction was performed, assuming metabolites are not sufficiently present in the blood in the first $2 \mathrm{~min}$ (9). The uniform recovery coefficient of 0.76 to correct for partial-volume effects in the myocardium was computed for the Biograph PET/CT scanner by assuming average myocardial thickness of $1 \mathrm{~cm}$, respiratory motion of $5 \mathrm{~mm}$ (10), average cardiac motion of $7.5 \mathrm{~mm}$ as established from our data, and intrinsic scanner spatial resolution of $4.8 \mathrm{~mm}$ (11).

Stress and rest flow values in $\mathrm{mL} / \mathrm{g} / \mathrm{mL}$ were computed for each sample on the polar map. To reduce noise in the curves, the interpolation was used and computations were performed in 70 myocardial regions with equal surface areas. For each of these regions, regional flows were computed. These calculations were then interpolated using a surface-area-weighted bicubic method to determine flow values for each polar map sample. MFR was computed by dividing each stress polar map sample by the rest samples at each point. The total MFR stress and rest flow was computed within the whole LV region bounded by the LV plane. The regional flow was then obtained by dividing the polar map into 3 regions (left anterior descending artery, left circumflex coronary artery, and right coronary artery) obtained from the standard 17-segment American Heart Association model (group option in QPET). Regional or segmental values were then provided to the user.

Syngo MBF Analysis. The cases were processed with some manual corrections of the contours as assessed by an experienced observer. Dynamic curves and polar maps were obtained for each sample of the polar map. A $1 \times 1 \times 1 \mathrm{~cm}$ volume of interest was automatically placed on the mitral valve plane to determine the LV input function. A previously developed model by Hutchins et al. (5) is used in syngo MBF. This is a 2-compartment model with 4 parameters. Similar to QPET, this model is considered irreversible since it assumes there is no reverse transport from metabolically bound compartment to the extravascular compartment. Partial-volume correction was performed internally within the model. Distribution volume was one of the parameters and was not assumed constant. All $10 \mathrm{~min}$ of the data were used for fitting. This model is described by first-order differential equations (5). The 4 parameters include vascular volume and 3 transport coefficients, which were computed by numeric optimization with the Levenberg-Marquardt method. Results were presented in polar map format for perfusion uptake, in $\mathrm{mL} / \mathrm{g} / \mathrm{min}$ for stress and rest flow values, and as MFR. Regional values for flow and MFR were provided for the 3 main coronary artery territories. The allocation of vascular territories was based on geometric division of the polar map, with a $150^{\circ}$ portion including the anterior and apical American Heart
Association segment regions designated as the left anterior descending artery territory and 2 consecutive clockwise $105^{\circ} \mathrm{seg}$ ments designated as the left circumflex coronary artery and right coronary artery territories (excluding the apical American Heart Association segment).

PMod Analysis. The PMod analysis required more manual operations, including volume-of-interest placement for the myocardium. After the images had been reoriented along the heart axis to form vertical long-axis, horizontal long-axis, and short-axis slices, regions of interest were drawn within the left ventricle on 6 consecutive image planes. These regions of interest were projected both onto the dynamic ${ }^{13} \mathrm{~N}$-ammonia images to generate blood time-activity curves (input function) and onto the right ventricle to correct for spillover of the septum. Similarly, myocardial regions of interest were drawn on the short-axis slices within the LV myocardium and were projected onto the dynamic images to obtain tissue activity curves. Time-activity curves were used to calculate mean MBF from the short-axis slices after 3 midventricular-slice myocardial regions of interest were assigned to the myocardial territories of the left anterior descending artery, left circumflex coronary artery, and right coronary artery. Both arterial and tissue activity curves were fitted to a previously validated tracer kinetic model to correct for spillover of activity from the blood pool into the left myocardium, providing values of regional and global MBF ( $\mathrm{mL} / \mathrm{min} / \mathrm{g})$. We used the standard kinetic modeling option in the PMod software, which assumes 1 compartment model when it is assumed that there is no metabolic trapping (3). Only the first $4 \mathrm{~min}$ after injection were used for the curve fitting.

\section{RESULTS}

Figure 1 shows linear regression results for QPET versus syngo MBF, QPET versus PMOD, and syngo MBF versus PMOD for flow values in $\mathrm{mL} / \mathrm{g} / \mathrm{min}$ combining stress and rest values $(n=66)$, with Bland-Altman plots. We noted that the outliers in this analysis have a high spillover fraction primarily in the right coronary artery. High spillover fractions may result in generally unreliable data, and they are accounted for differently in QPET (12). Therefore, we evaluated regressions and SEs after excluding cases with increasing spillover fractions. The spillover fractions are reported in the output of QPET (Table 3). The ranges of spillover fractions were $0.18-0.62$ for the left anterior descending artery, 0.11-0.59 for the left circumflex coronary artery, and 0.160.73 for the right coronary artery. On the basis of this analysis, we excluded the cases with a high spillover fraction $(>65 \%)$ in the right coronary artery region $(n=5: 4$ stress and 1 rest in 4 patients). Three of these patients belonged to the very low likelihood group, and hence the final nonischemic population consisted of 15 patients and the final ischemic population consisted of 14 patients. Figure 2 shows the corresponding linear regression results, with Bland-Altman plots for the remaining 61 studies with an acceptable spillover fraction (29 stress and 32 rest).

On the basis of these findings, further analysis was performed with studies in which the spillover fraction was below 65\%. Figure 3 shows regression and Bland-Altman plots for MFR values for QPET, syngo MBF, and PMOD. When rest scans were analyzed separately using the Bland- 


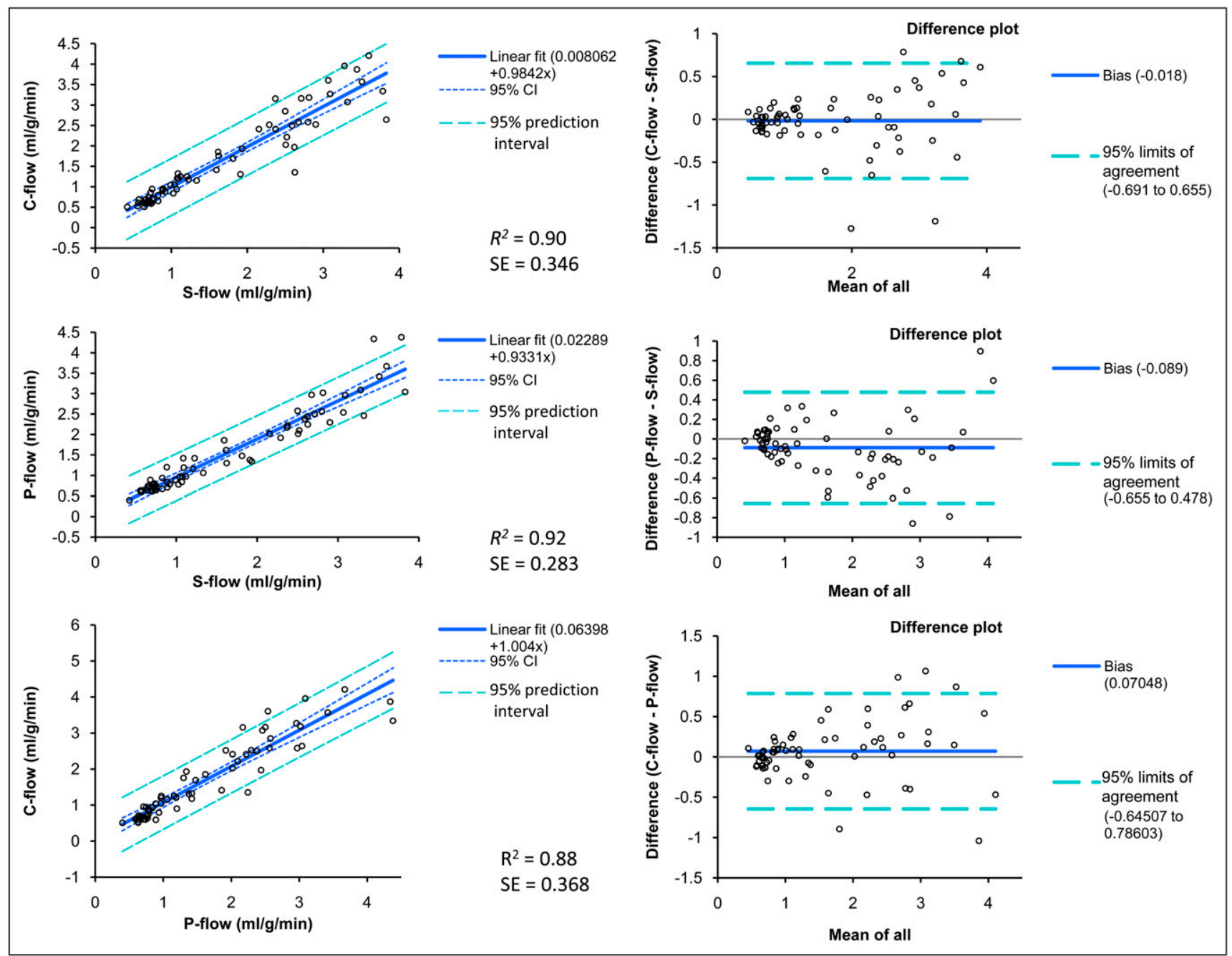

FIGURE 1. Linear regression (left) and Bland-Altman (right) plots for QPET vs. syngo MBF, QPET vs. PMOD, and syngo MBF vs. QPET for flow values combining stress and rest values $(n=66)$. C $=$ QPET; P $=$ PMOD; $S=$ syngo MBF.

Altman methods, the $95 \%$ confidence limits for the resting flow were -0.22 to $0.21 \mathrm{~mL} / \mathrm{g} / \mathrm{min}$ (bias $=0.03$ ) for QPET versus syngo $\mathrm{MBF},-0.28$ to $0.24 \mathrm{~mL} / \mathrm{g} / \mathrm{min}$ (bias $=-0.02$ ) for syngo MBF versus PMOD, and -0.27 to $0.24 \mathrm{~mL} / \mathrm{g} / \mathrm{min}$ (bias $=-0.02$ ) for QPET versus PMOD. In addition, in Table 4 we summarize the mean absolute differences be- tween the 3 methods for rest flow, stress flow, and MFR values. Figure 4 shows the regression plots and SEs between each of these methods in each of the vascular territories.

Because these 3 tools were evaluated by different observers, we also evaluated the interobserver reproducibility for each tool. The results are presented in Table 5.

TABLE 3

Regression $R^{2}$ and SE Among the 3 Methods When Cases with High Spillover Fraction Are Excluded

\begin{tabular}{|c|c|c|c|c|c|}
\hline \multirow[b]{2}{*}{ Spillover fraction threshold } & \multirow[b]{2}{*}{$N$} & \multicolumn{2}{|c|}{$R^{2}$} & \multicolumn{2}{|c|}{ SE } \\
\hline & & QPET vs. syngo MBF & QPET vs. PMOD & QPET vs. syngo MBF & QPET vs. PMOD \\
\hline All & 66 & 0.90 & 0.88 & 0.346 & 0.368 \\
\hline$<0.70$ & 64 & 0.92 & 0.91 & 0.307 & 0.319 \\
\hline$<0.65$ & 61 & 0.95 & 0.92 & 0.250 & 0.311 \\
\hline$<0.60$ & 56 & 0.96 & 0.92 & 0.222 & 0.305 \\
\hline$<0.5$ & 43 & 0.95 & 0.93 & 0.230 & 0.274 \\
\hline
\end{tabular}




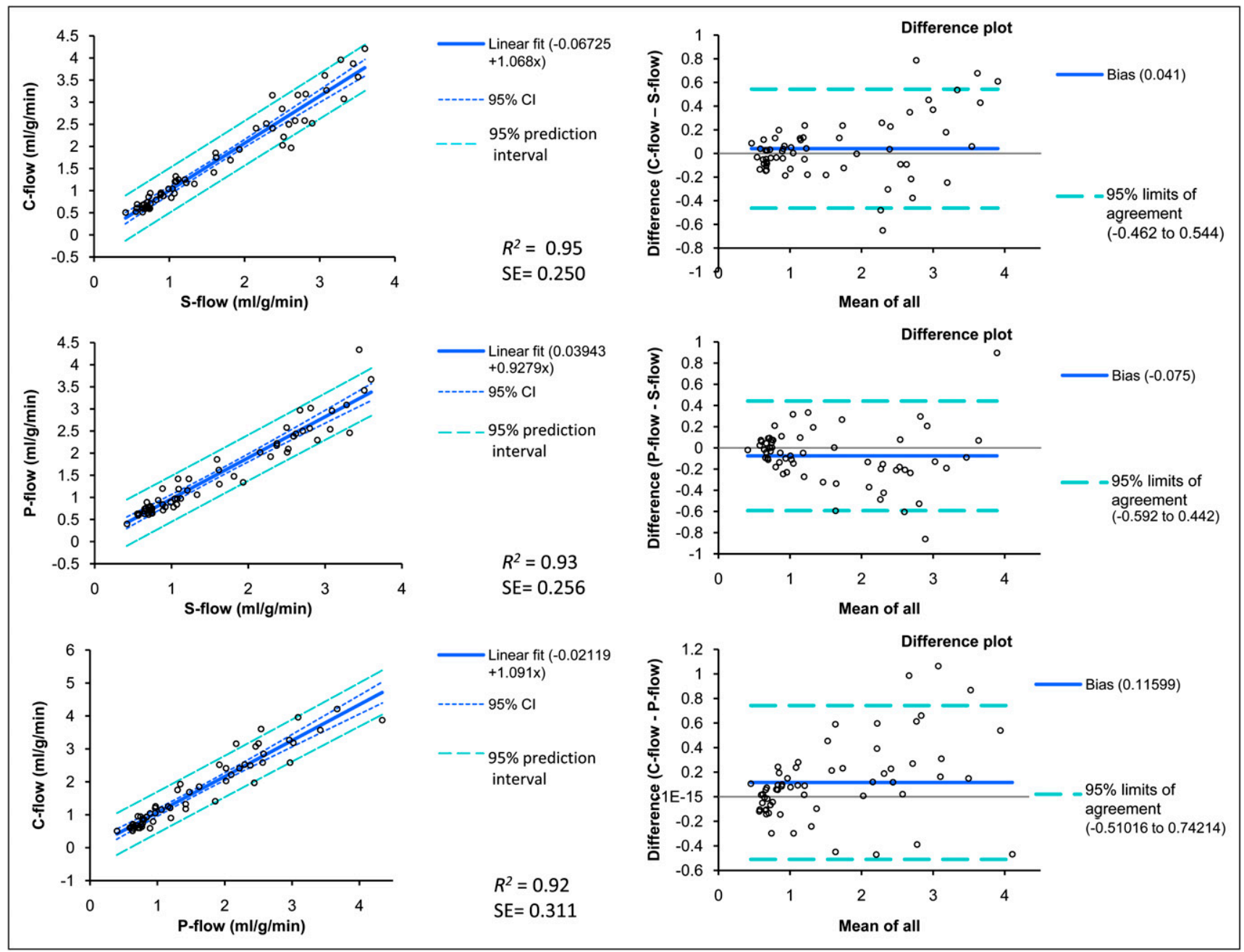

FIGURE 2. Linear regression (left) and Bland-Altman (right) plots for QPET vs. syngo MBF, QPET vs. PMOD, and syngo MBF vs. QPET for flow values combining stress and rest values $(n=61)$ after excluding 5 cases with high spillover fraction $(>0.65)(n=61)$. C $=$ QPET; P = PMOD; $\mathrm{S}=$ syngo MBF.

We separately evaluated the findings in the very low likelihood group $(n=15)$ and in the ischemic group $(n=$ 14). Global stress flow, rest flow, and MFR values were consistent among all 3 methods $(P=$ not statistically significant, 1-way ANOVA of repeated measures) in the very low likelihood and ischemic groups. In addition, 1-way ANOVA of repeated measures did not show any differences between values in any of the vascular regions. Mean values and ranges for global stress-rest flow and MFR are shown separately in Table 6 for the very low likelihood and ischemic groups. Mean normal MFR values were similar for QPET, syngo MBF, and PMOD (3.39 $\pm 1.22,3.41 \pm$ 0.76 , and $3.66 \pm 1.19$, respectively) by 1 -way ANOVA $(P=0.74)$. The reference ranges agree with previous reference ranges published for this tracer (4). In Figure 5, we show the regional stress-rest flow (Fig. 5A) and MFR values (Fig. 5 B) in each of the vascular territories for very low likelihood and ischemic patients.
Figure 6 illustrates the results obtained by the 3 methods for a 65 -y-old man. All methods show abnormal MFR in the 3 vascular territories.

\section{DISCUSSION}

Obtaining absolute flow measurements from dynamic PET studies is of considerable interest since it can potentially overcome the problems related to the relative normalization of static perfusion images, which can mask significant coronary artery disease (13). In addition, dynamic flow can be used to study other diseases such as microvascular dysfunction $(14,15)$. For clinical use, reliable tools for the fully automated computation of global and regional blood flow are needed (16). Several tools for absolute flow quantification have recently been developed and are available for clinical or research use (2). They combine advances in the automated detection of the myocardium with established kinetic modeling techniques and allow 


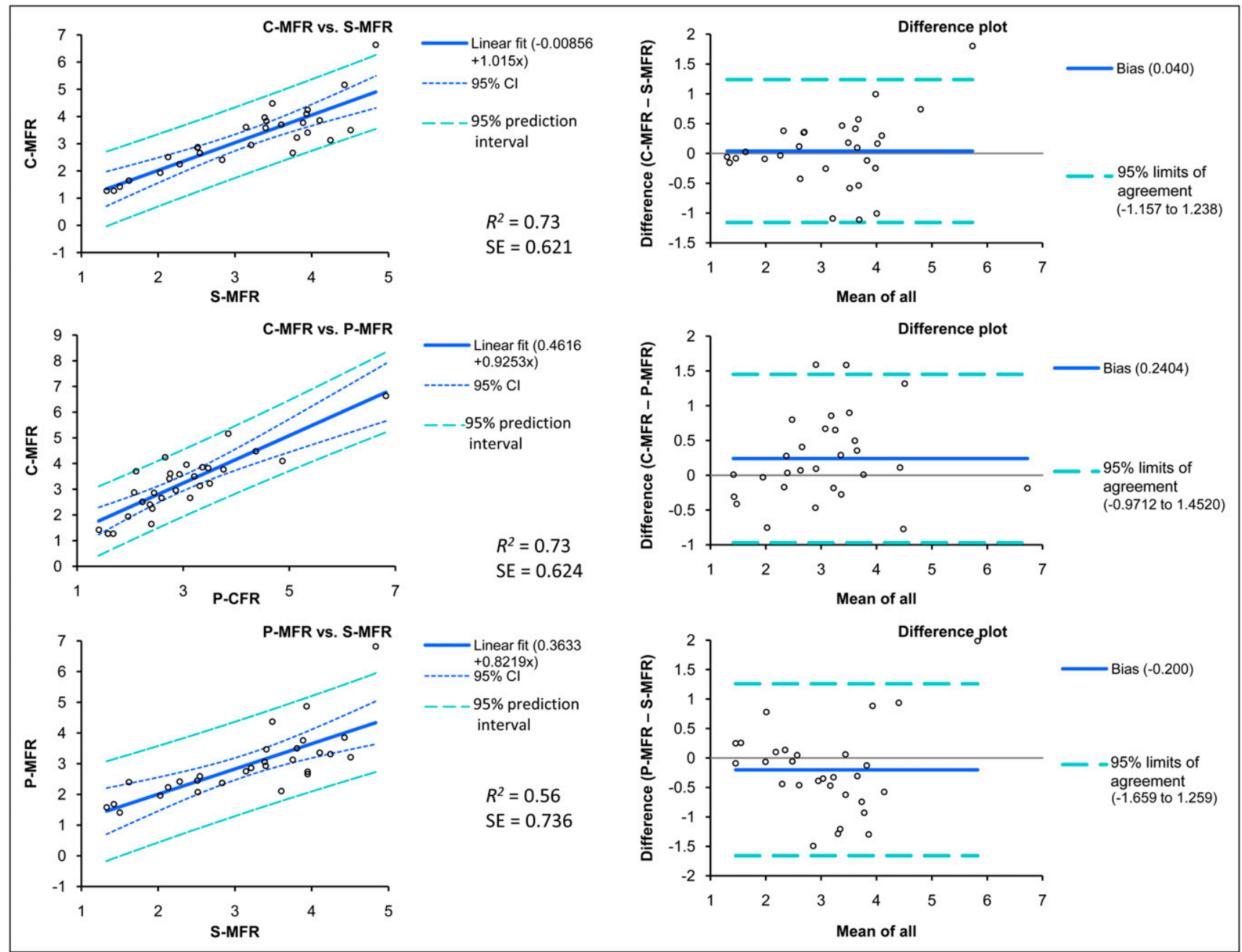

FIGURE 3. MFR. Linear regression (left) and Bland-Altman (right) plots for QPET vs. syngo MBF, QPET vs. PMOD, and PMOD vs. syngo MBF $(n=29)$. C $=$ QPET; P $=$ PMOD; $\mathrm{S}=$ syngo MBF.

rapid and automated analysis of dynamic scans. Furthermore, recent advances in PET/CT systems provide acquisition of list-mode data (1), allowing reformatting of PET scan data into dynamic, static, or gated frames as required and obviating multiple acquisitions. Therefore, the capabilities of absolute flow measurements have the potential of becoming more widely utilized in clinical practice and not just in academic centers (17). Invariably, for clinical use, the results obtained at different laboratories with different methods will need to be compared, and appropriate reference limits will need to be established. To provide a basis for such comparisons, we directly evaluated 3 existing clinical implementations of the kinetic modeling packages for the computation of regional stress and rest flow and myocardial perfusion reserve for ${ }^{13} \mathrm{~N}$-ammonia. Each method uses a distinct kinetic model, either 1 or 2 compartments. In addition, the samples obtained from the myocardium and blood pool as input to the model are derived with different approaches. QPET and syngo MBF are automated and PMOD relies on more time-consuming operator delineation of myocardium and blood pool. Although the kinetic modeling methods used in these tools were validated in separate studies for stand-alone PET, previous research in this area focused on the development of kinetic modeling without automated detection of the left ventricle and blood pool (9). Furthermore, previous evaluations of the kinetic ${ }^{13} \mathrm{~N}-$

TABLE 4

Mean Absolute Differences ( \pm SD) Between Measurements Obtained by the 3 Methods

\begin{tabular}{lccr}
\hline \multicolumn{1}{c}{ Comparison } & Rest flow $(\mathrm{mL} / \mathrm{g} / \mathrm{min})$ & Stress flow $(\mathrm{mL} / \mathrm{g} / \mathrm{min})$ & MFR \\
\hline QPET vs. syngo MBF & $0.09 \pm 0.06$ & $0.28 \pm 0.22$ & $0.44 \pm 0.42$ \\
PMOD vs. syngo MBF & $0.11 \pm 0.08$ & $0.30 \pm 0.22$ & $0.58 \pm 0.50$ \\
QPET vs. PMOD & $0.11 \pm 0.08$ & $0.39 \pm 0.27$ & $0.49 \pm 0.44$ \\
\hline
\end{tabular}




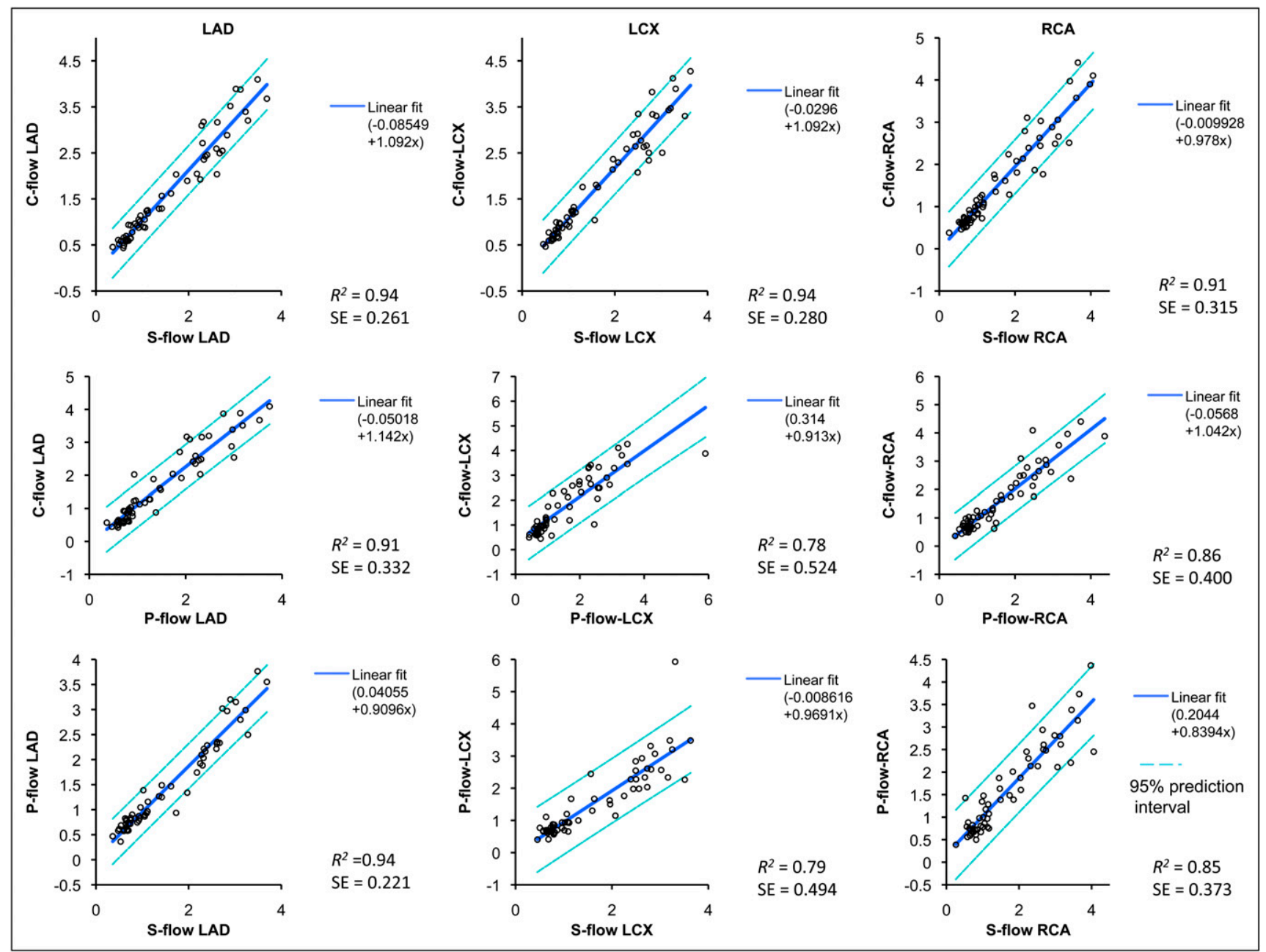

FIGURE 4. Regional flow regression plots among the 3 methods $(n=61)$. All flow values are reported in $\mathrm{mL} / \mathrm{g} / \mathrm{min}$. C $=\mathrm{QPET} ; \mathrm{P}=\mathrm{PMOD}$; $\mathrm{S}=$ syngo MBF.

ammonia software were performed for stand-alone PET scanners (18), and no such comparisons have been obtained to date for PET/CT data. In addition, comparisons were not previously performed for PET/CT data acquired in 3D mode.

Despite different assumptions in the models, different methods of finding and segmenting the myocardium and blood pool automatically by QPET and syngo MBF and manually by PMOD, and different methods of extracting the input and myocardial curves, the results demonstrate excellent agreement among the methods (Fig. 1). We did identify a reason for the discrepancies, namely a significant spillover of blood-pool activity into the myocardium. Eliminating the 5 datasets with high spillover decreased the confidence limits significantly (Fig. 2). Regional agreement was also good in all territories (Fig. 4). Regionally, the 95\% confidence limits decreased significantly in the right coronary artery territory after elimination of the high-spillover cases. This decrease is most likely due to the fact that the spillover is modeled differently in these packages and that,

TABLE 5

$R^{2}$, SE, Interobserver Reproducibility, and 95\% Confidence Interval (Cl) for the 3 Methods

\begin{tabular}{|c|c|c|c|c|c|c|c|c|}
\hline \multirow[b]{2}{*}{ Method } & \multicolumn{4}{|c|}{ Flow (mL/g/min) } & \multicolumn{4}{|c|}{ MFR } \\
\hline & $R^{2}$ & SE & Bias & $\mathrm{Cl}$ & $R^{2}$ & SE & Bias & $\mathrm{Cl}$ \\
\hline QPET & 0.99 & 0.09 & -0.04 & $-0.26,-0.19$ & 0.97 & 0.20 & -0.06 & $-0.43,-0.31$ \\
\hline Syngo MBF & 0.99 & 0.12 & 0.04 & $-0.2,0.27$ & 0.91 & 0.29 & 0.19 & $-0.38,0.76$ \\
\hline PMOD & 0.99 & 0.10 & 0.03 & $-0.16,0.22$ & 0.94 & 0.23 & -0.07 & $-0.56,0.42$ \\
\hline
\end{tabular}


TABLE 6

Stress and Rest Flow and MFR Results Obtained in Very Low Likelihood and Ischemic Patients by the 3 Methods

\begin{tabular}{llll}
\hline \multicolumn{1}{c}{ Group } & Stress flow $(\mathrm{mL} / \mathrm{g} / \mathrm{min})$ & Rest flow $(\mathrm{mL} / \mathrm{g} / \mathrm{min})$ & MFR \\
\hline Very low likelihood $(n=15)$ & & & $3.66 \pm 1.19(2.25-6.63)$ \\
QPET & $3.00 \pm 0.66(1.97-4.21)$ & $0.87 \pm 0.24(0.59-1.25)$ & $3.41 \pm 0.76(2.13-4.83)$ \\
Syngo MBF & $2.89 \pm 0.45(2.29-3.60)$ & $0.90 \pm 0.20(0.68-1.33)$ & $3.39 \pm 1.22(2.23-6.82)$ \\
PMOD & $2.77 \pm 0.65(1.92-4.34)$ & $0.85 \pm 0.16(0.69-1.40)$ & \\
Ischemic $(n=14)$ & & & \\
QPET & $2.04 \pm 0.80(0.90-3.60)$ & $0.78 \pm 0.24(0.51-1.32)$ & $2.71 \pm 1.00(1.27-3.76)$ \\
Syngo MBF & $1.98 \pm 0.66(0.88-3.07)$ & $0.74 \pm 0.21(0.42-1.09)$ & $2.90 \pm 1.17(1.33-4.51)$ \\
PMOD & $1.83 \pm 0.57(1.16-3.02)$ & $0.77 \pm 0.23(0.40-1.42)$ & $2.51 \pm 0.74(1.41-3.76)$
\end{tabular}

Data are mean $\pm S D$, with ranges in brackets.

above certain thresholds, these models become unreliable. In addition, patient motion could make these spillover assessments inconsistent through the frames. Patient motion during a dynamic scan could also result in attenuation correction errors for some of the frames and, consequently, incorrect blood flow measurements (19). This effect, however, requires additional study.

In this study, we have experimentally established a threshold of $65 \%$ as a reasonable cutoff for the regional spillover fraction of counts from blood pool to myocardium. High spillover fractions were observed mostly in the right coronary artery territory ( $4 / 5$ patients). The remaining patient had a spillover higher than $65 \%$ in the left circumflex coronary artery territory. The reason for the high spillover fraction in the observed patients was likely the variable location of the myocardium during the dynamic scan due to patient motion and consequently contamination of the myocardium with blood-pool activity as visually observed. In phantom evaluations of spillover fractions, much lower values were obtained (20). In practical terms, the high spillover fraction remains a limitation for clinical analysis since the results may not be reliable in such cases, as demonstrated here. Although all 3 packages correct for spillover, the models seem to diverge at this high-spillover fraction. Ideally, a better spillover correction method should be designed to avoid such discrepancies. However, for the

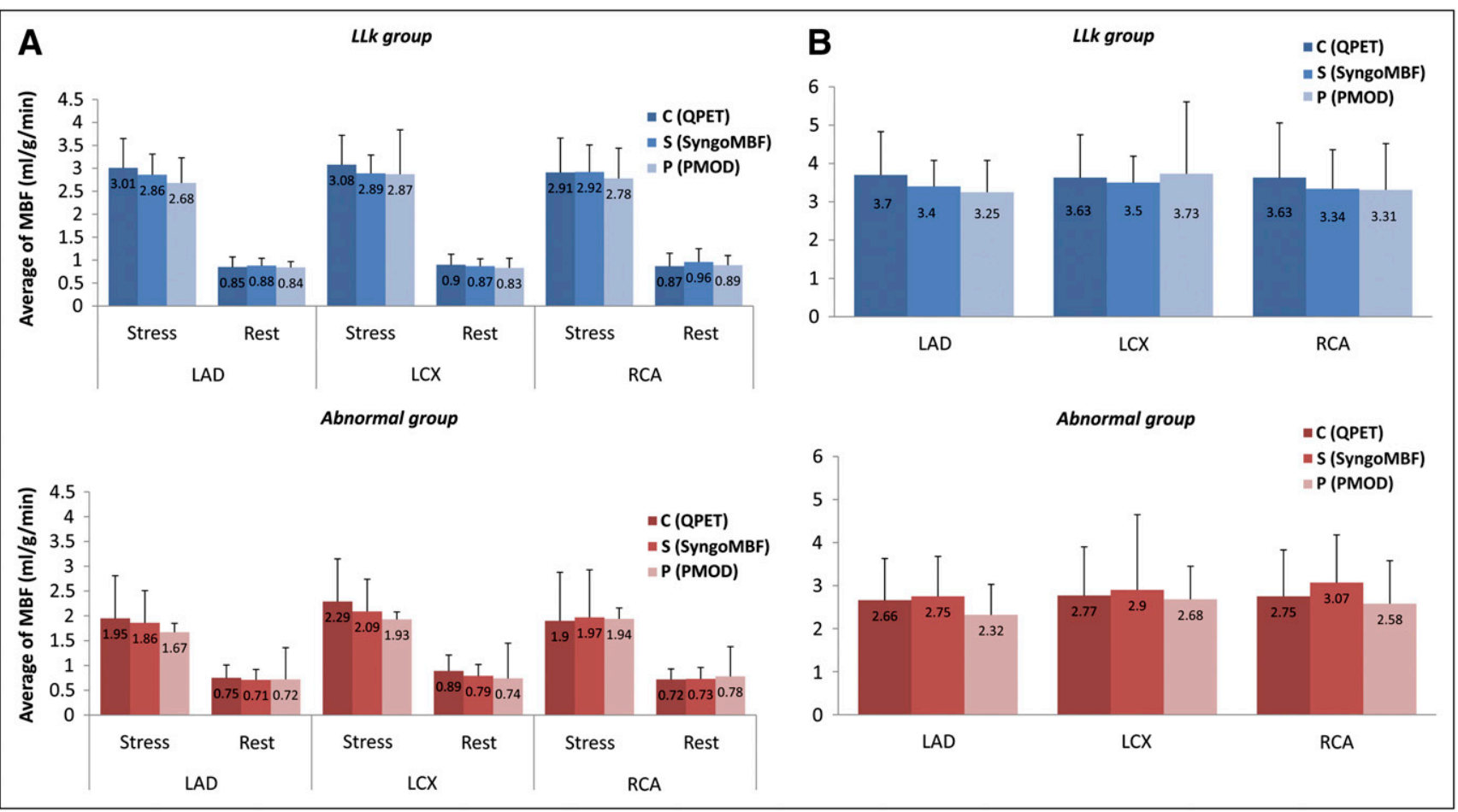

FIGURE 5. Mean regional stress and rest flow MBF (A) and MFR (B) results obtained in very low likelihood and ischemic patient groups by the 3 methods. None of the results were significantly different from one another (1-way ANOVA of repeated measures) in either very low likelihood or ischemic group. 


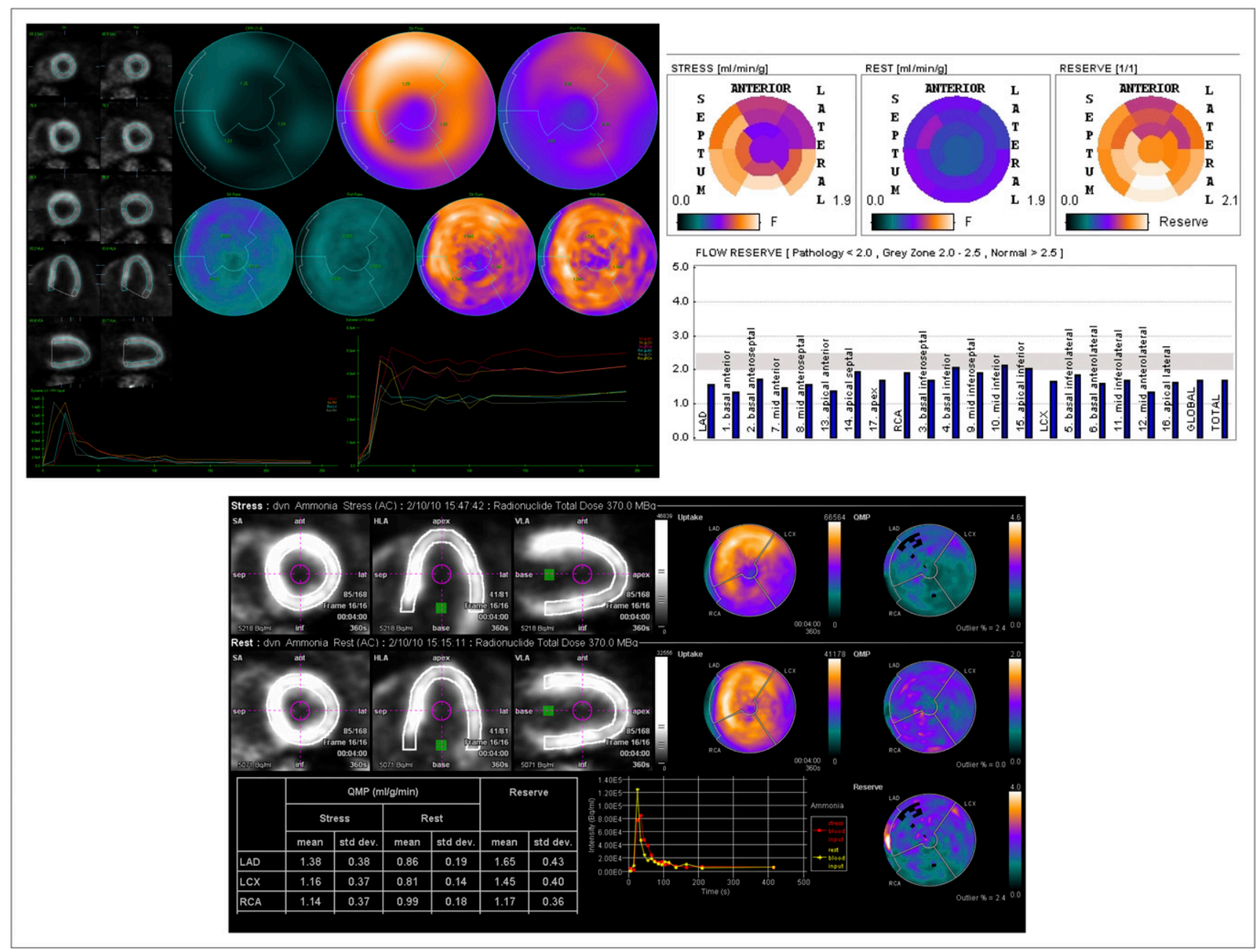

FIGURE 6. Example output generated by QPET (top left), PMOD (top right), and syngo MBF (bottom) in 65-y-old man. All 3 methods demonstrated abnormal MFR (QPET: stress flow $=1.20 \mathrm{~mL} / \mathrm{g} / \mathrm{s}$, rest flow $=0.96 \mathrm{~mL} / \mathrm{g} / \mathrm{s}, \mathrm{MFR}=1.29 ;$ syngo $\mathrm{MBF}$ : stress flow $=1.23 \mathrm{~mL} / \mathrm{g} / \mathrm{min}$, rest flow $=0.89 \mathrm{~mL} / \mathrm{g} / \mathrm{min}, \mathrm{MFR}=1.42$; PMOD: stress flow $=1.42 \mathrm{~mL} / \mathrm{g} / \mathrm{min}$, rest flow $=0.84 \mathrm{~mL} / \mathrm{g} / \mathrm{min}, \mathrm{MFR}=1.68$ ).

time being, even flagging of such questionable cases by the quantitative software (by providing spillover fraction values) can provide a warning to the clinicians. Though not an ideal solution, it is a step toward identifying the potential problem.

Motion correction software could potentially eliminate this effect. However, such software would have to also eliminate motion between the PET and the CT scans to avoid attenuation correction errors on some frames due to PET/CT misregistration (21). To date, none of the packages studied in this work provide such a capability. Furthermore, there is currently no evidence that such algorithms would actually improve the quantitative flow results.

Despite a good correlation of stress and rest flow values and MFR among the 3 implementations, the 95\% confidence limits and SE estimates remain somewhat high (Figs. 2 and 3). However, as is evident from the Bland-Altman plots, the discrepancies occur for the higher values (above reference values), and there is no evident bias; hence, they are not likely to be clinically important. As shown in Table 5 , interobserver reproducibility within each tool is not negligible, and a significant component of the intersoftware variability could be attributed to interobserver variability since data were analyzed by different observers for each tool. When SE estimates are compared, this interobserver variation can account for $30 \%-46 \%$ of the variability in intersoftware comparisons. The interobserver reproducibility reported here is consistent with previously reported results (2). As seen in Table 6, the mean reference ranges of stress and rest flow, as well as for myocardial perfusion reserve, are not significantly different for the 3 different tools. The results obtained by QPET and PMOD show more heterogeneity at the upper level of stress flows and MFR, likely because of internal constraints used by syngo MBF. This variation does not affect the lower reference ranges for these tools, which are comparable. Furthermore, regional analysis of reference values reveals no significant differences between any of these tools in any region (Fig. 5), 
despite the fact that regional definitions were slightly different among the methods. In addition, there are no differences between the regions for each of the tools. The mean reference ranges for all 3 tools agree with previously published data for normal flows $(9,22)$.

The previously established lower normal limit of 2.0-2.5 $\mathrm{mL} / \mathrm{g} / \mathrm{min}$ for stress flow of ${ }^{15} \mathrm{O}$ water (23) agrees with the results obtained in this study for ${ }^{13} \mathrm{~N}$-ammonia. Therefore, it should be possible to establish absolute (tool- and regionindependent) reference limits for the stress and rest flows and values, greatly simplifying interpretation of the dynamic finding for clinicians. Further refinement, however, may be needed for precise absolute normal flow limits, with the correction of resting rate-pressure product or age of the subject, since these factors are known to influence the resting blood flow and coronary flow reserve (24).

This study had several limitations. The gold standard for MBF was unavailable. The goal, however, was to compare the results from the clinical implementations of the kinetic modeling software tools. In this work, we studied only ammonia blood flow. A separate study will be needed for the more prevalent ${ }^{82} \mathrm{Rb}$ dynamic flow analysis, which uses other methods of kinetic modeling. However, current implementations of ${ }^{82} \mathrm{Rb}$ analysis methods have been calibrated by definition to the ${ }^{13} \mathrm{~N}$-ammonia results (22). Although QPET and syngo MBF performed automated analysis of the dynamic flow, some user intervention (contour adjustment) was still required in a minority of cases, and consequently some variability of the results occurs as shown in our results in Table 5. Although we have evaluated the data analyzed with different software tools, the data were obtained with 1 scanner and 1 imaging and reconstruction protocol. It is possible that there may be discrepancies in reference ranges due to the scanner type, reconstruction, and dynamic protocols applied; this will require further study. The sample size in our study was relatively small. Future studies should have a larger sample size with more precisely defined and known cardiovascular risk factors. Patients within our ischemic group included those with several risk factors. These risk factors may affect MFR values. However, the main goal of this study was to compare the clinical tools for both normal and abnormal flows. The actual clinical value of the MFR and flow measurements will need to be established in a separate study.

\section{CONCLUSION}

Different implementations of 1- and 2-compartment models for dynamic ${ }^{13} \mathrm{~N}$-ammonia PET demonstrate excellent correlation in MBF and MFR for each vascular territory, with similar mean MFR values and similar flow values. Reference limits for stress and rest flow and MFR were established for the 3D PET/CT scanner. These reference limits appear to be interchangeable between different methods of analysis. Blood-to-myocardium spillover fraction, especially in the right coronary artery territory, should be monitored during the quality control of the dynamic data.

\section{DISCLOSURE STATEMENT}

The costs of publication of this article were defrayed in part by the payment of page charges. Therefore, and solely to indicate this fact, this article is hereby marked "advertisement" in accordance with 18 USC section 1734.

\section{ACKNOWLEDGMENT}

We would like to thank Arpine Oganyan for editing and proofreading the text. Some others from Cedars-Sinai Medical Center receive royalties for the licensure of software used in the quantitative assessment of function, perfusion, and viability, a portion of which is distributed to some of the authors of this article. Dr. Ludovic Le Meunier is an employee of Siemens Medical Systems, PET Division. No other potential conflict of interest relevant to this article was reported.

\section{REFERENCES}

1. Di Carli MF, Dorbala S, Meserve J, El Fakhri G, Sitek A, Moore S. Clinical myocardial perfusion PET/CT. J Nucl Med. 2007;48:783-793.

2. Klein R, Renaud J, Ziadi $\mathrm{M}$, et al. Intra- and inter-operator repeatability of myocardial blood flow and myocardial flow reserve measurements using rubidium-82 pet and a highly automated analysis program. J Nucl Cardiol. 2010;17:600-616.

3. DeGrado TR, Hanson M, Turkington T, Delong D, Brezinski D, Vallee J. Estimation of myocardial blood flow for longitudinal studies with ${ }^{13} \mathrm{~N}$-labeled ammonia and positron emission tomography. J Nucl Cardiol. 1996;3:494-507.

4. Choi Y, Hawkins S-C, Hawkins R, et al. A simplified method for quantification of myocardial blood flow using nitrogen-14 ammonia and dynamic PET. J Nucl Med. 1993;34:488-497.

5. Hutchins GD, Schwaiger M, Rosenspire K, Krivokapich J, Schelbert H, Kuhl D. Noninvasive quantification of regional blood flow in the human heart using N-13 ammonia and dynamic positron emission tomographic imaging. J Am Coll Cardiol. 1990;15:1032-1042.

6. Wilson PW, D'Agostino R, Levy D, Belanger A, Silbershatz H, Kannel W. Prediction of coronary heart disease using risk factor categories. Circulation. 1998;97:1837-1847.

7. Slomka P, Germano G, Kavanagh P, Javadi M, Berman D, Bengel F. Evaluation of a new automatic algorithm for quantification of ECG-gated ${ }^{82} \mathrm{Rb}$ cardiac PET [abstract]. J Nucl Med. 2009;50(suppl 2):217P.

8. Germano G, Kiat H, Kavanagh P, et al. Automatic quantification of ejection fraction from gated myocardial perfusion SPECT images. $J$ Nucl Med. 1995;36:2138-2147.

9. Choi Y, Hawkins S-C, Hawkins R, et al. Quantification of myocardial blood flow using N-13-ammonia and PET: comparison of tracer models. J Nucl Med. 1999;40:1045-1055.

10. Martinez-Möller A, Zikic D, Botnar R, et al. Dual cardiac-respiratory gated PET: implementation and results from a feasibility study. Eur J Nucl Med Mol Imaging. 2007;34:1447-1454.

11. Townsend D, Jakoby B, Long MJ, et al. Performance and clinical workflow of a new combined PET/CT scanner [abstract]. J Nucl Med. 2007;48(suppl 2):437P.

12. Choi Y, Hawkins R, Huang S. Parametric images of myocardial metabolic rate of glucose generated from dynamic cardiac PET and 2-[ $\left.{ }^{18} \mathrm{~F}\right]$ fluoro-2-deoxy-d-glucose studies. J Nucl Med. 1991;32:733-738.

13. Parkash R, DeKemp R, Ruddy T, et al. Potential utility of rubidium 82 PET quantification in patients with 3-vessel coronary artery disease. J Nucl Cardiol. 2004;11:440-449.

14. Cecchi F, Olivotto L, Gistri R, Lorenzoni R, Chiriatti G, Camici P. Coronary microvascular dysfunction and prognosis in hypertrophic cardiomyopathy. $N$ Engl J Med. 2003;349:1027-1035.

15. Schindler TH, Schelbert H, Quercioli A, Dilsizian V. Cardiac PET imaging for the detection and monitoring of coronary artery disease and microvascular health. JACC Cardiovasc Imaging. 2010;3:623-640.

16. Camici PG, Rimoldi O. The clinical value of myocardial blood flow measurement. J Nucl Med. 2009;50:1076-1087. 
17. Beanlands RS, Ziadi M, Williams K. Quantification of myocardial flow reserve using positron emission imaging the journey to clinical use. J Am Coll Cardiol. 2009;54:157-159.

18. El Fakhri G, Kardan A, Dorbala S, et al. Reproducibility and accuracy of quantitative myocardial blood flow assessment with ${ }^{82} \mathrm{Rb}$ PET: comparison with ${ }^{13} \mathrm{~N}$ ammonia PET. J Nucl Med. 2009;50:1062-1071.

19. Klein R, Beanlands R, DeKemp R. Quantification of myocardial blood flow and flow reserve: technical aspects. $J$ Nucl Cardiol. 2010;17:555-570.

20. Nuyts H, Maes A, Vrolix M, et al. Three-dimensional correction for spillover and recovery of myocardial PET images. J Nucl Med. 1996;37: $767-774$.
21. Slomka PJ, Le Meunier L, Hayes S, et al. Comparison of myocardial perfusion ${ }^{82} \mathrm{Rb}$ PET performed with CT- and transmission CT-based attenuation correction. J Nucl Med. 2008;49:1992-1998.

22. Lortie M, Beanlands R, Yoshinaga K, Klein R, Dasilva J, DeKemp RA. Quantification of myocardial blood flow with ${ }^{82} \mathrm{Rb}$ dynamic PET imaging. Eur J Nucl Med Mol Imaging. 2007;34:1765-1774.

23. Kajander S, Joutsiniemi E, Saraste M, et al. Cardiac positron emission tomography/ computed tomography imaging accurately detects anatomically and functionally significant coronary artery disease. Circulation. 2010;122:603-613.

24. Czernin J, Muller P, Chan SB, et al. Influence of age and hemodynamics on myocardial blood flow and flow reserve. Circulation. 1993;88:62-69. 